\title{
Real-Time Electron Counting for Continuous TEM Imaging of Sensitive Samples
}

\author{
Ben Miller ${ }^{1 *}$, Anahita Pakzad ${ }^{1}$ and Stephen Mick ${ }^{1}$ \\ 1. Gatan Inc. Pleasanton, CA, USA \\ * Corresponding author: BMiller@gatan.com
}

In situ transmission electron microscopy (TEM) enables observation of sample dynamics at the nanoscale. However, observations performed in the TEM have always suffered from various deleterious effects of the electron beam, and these are compounded if the sample is continuously imaged over time. These effects are not always understood or accounted for and can make it impossible to image certain classes of materials. Efforts to mitigate electron beam effects have traditionally involved limiting electron dose, but this often comes at the expense of limiting spatial/temporal resolution, or the duration of observation.

Recent advances in camera technology have enabled breakthroughs for cryo-electron microscopy (cryoEM) - a low electron dose TEM technique commonly used with sensitive biological samples. Today, at low electron doses, nearly every electron that passes through a sample can be individually identified and counted. The counted images that result are of unprecedented quality [1]. This electron counting technology can be applied to the study of sample dynamics in materials science to reduce the impact of the electron beam, thereby both enabling the observation of the fundamental structure of materials such as metal-organic frameworks (MOFs) and extending the duration and resolution of continuous observations of beam-sensitive specimens.

The data shown in Figure 1 were acquired with Gatan counting cameras on three different TEMs. Figure 1A was acquired from a ZIF-8 MOF using a K2 ${ }^{\circledR}$ camera in electron counting mode on an FEI Titan $80-$ 300 TEM at KAUST [2]. Figure 1B was acquired from a detonation nano-diamond sample using a K ${ }^{\mathrm{TM}}$ IS electron counting camera on a JEOL F200 TEM at Gatan; a DENSsolutions Wildfire holder was used to heat the nano-diamond sample. Figure $1 \mathrm{C}$ was acquired from a $\mathrm{Cu}-\mathrm{Sn}$ alloy using a $\mathrm{K} 3 \mathrm{IS}$ electron counting camera on an FEI Tecnai TEM at Gatan; a Protochips Fusion holder was used to heat this sample.

Figure 1A shows the accumulation of electron counts in a single image of a MOF sample over three seconds. Using older cameras, it was impossible to image MOFs at high spatial resolution, as these samples are quickly destroyed by the electron beam. Figure 1B shows drift-corrected images of a nanodiamond heated to $1000{ }^{\circ} \mathrm{C}$ with $1.6 \mathrm{~s}$ total exposure times and fast Fourier transforms (FFT) were taken from the blue regions. The images were obtained by averaging 16 consecutive frames from continuous counted videos captured at $10 \mathrm{fps}$ for 30 minutes. The first video was acquired with a low dose rate of 100 $\mathrm{e}^{-/} / \AA^{2} / \mathrm{s}$, and little change was observed in the nano-diamond sample over 30 minutes. The second video was acquired immediately following the first, with a dose rate of $1000 \mathrm{e}^{-/} \AA^{2} / \mathrm{s}$; significant conversion of diamond to graphitic layers was observed at this higher dose rate - a dose rate that has been typical for the acquisition of low-dose high-resolution TEM images [3]. Figure 1C shows several frames from a counted in-situ TEM video. A Cu-Sn alloy (10:90) was heated to $600{ }^{\circ} \mathrm{C}$, at which temperature the alloy dissociates, leaving both small and large particles of pure $\mathrm{Sn}$. The video, acquired during the temperature ramp, was 2 min long and captured at 20 frames per second, but the dose rate was just $27 \mathrm{e}^{-} / \AA^{2} / \mathrm{s}$ and the total electron dose was only $3240 \mathrm{e}^{-} / \AA^{2}$. A traditional TEM camera (e.g. Gatan Ultrascan $\AA$ ) would likely require at least $5 \mathrm{x}$ this dose rate to achieve a similar result [4]. 


\section{References:}

[1] X Li et al., Nature Methods 10 (2013), p. 584.

[2] Y Zhu et al., Nature Materials 16 (2017), p. 532.

[3] S Helveg et al., Micron 68 (2015), p. 176.

[4] The nano-diamond sample used in this work was kindly provided by Dr. Shery Chang (ASU)

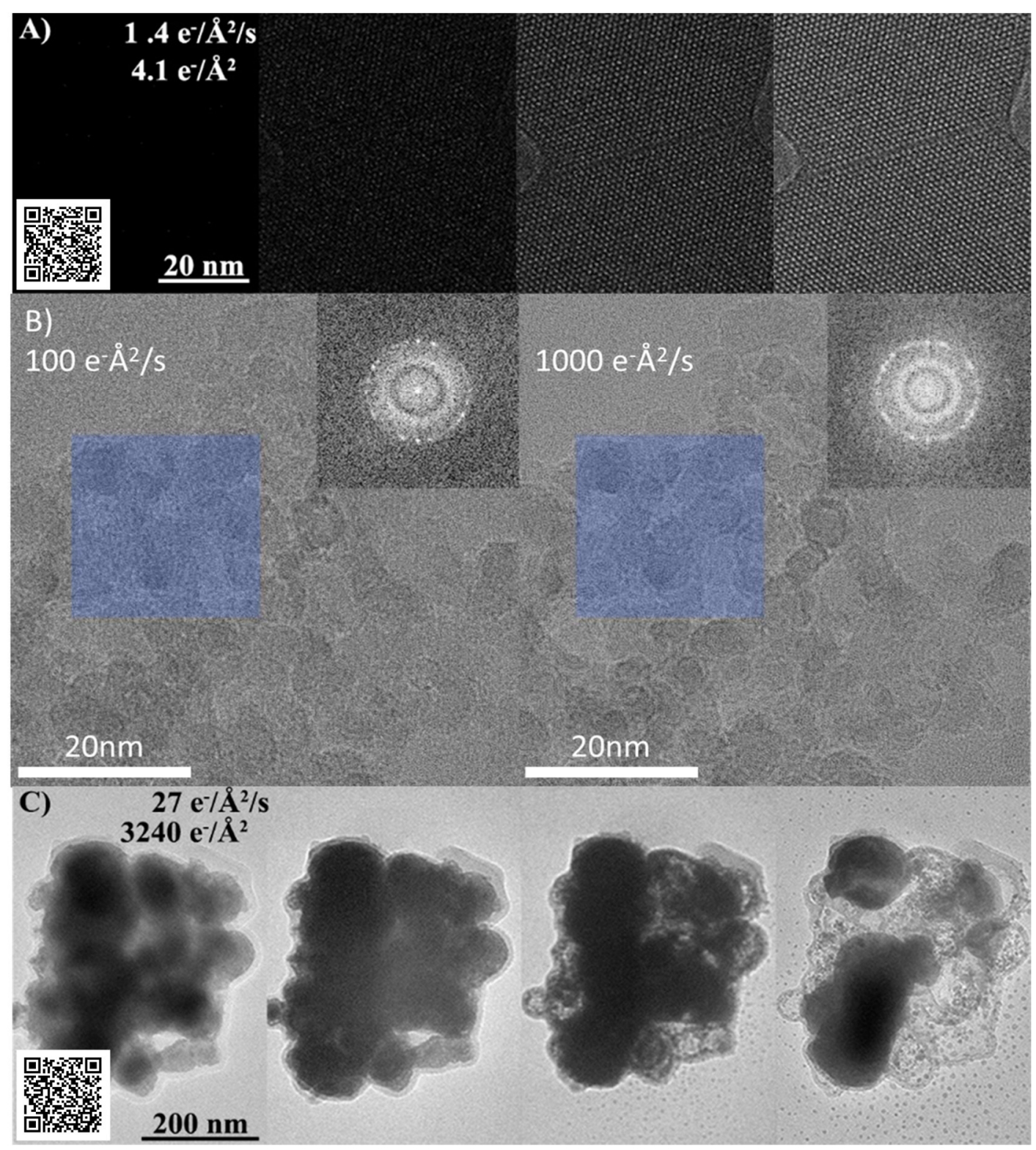

Figure 1. Data showing low-dose counted images/video from several samples. A) A single image of a MOF sample [2] is built up over 3 seconds. B) Comparison of low and high dose rate images from in-situ TEM videos of a nano-diamond sample heated to $1000^{\circ} \mathrm{C}$. C) Selected frames from a low-dose in-situ $\mathrm{TEM}$ video of $\mathrm{Cu}-\mathrm{Sn}$ alloy dissociation and $\mathrm{Sn}$ nanoparticle formation. QR codes link to full videos. 